УДК 621.315 .592

\title{
Исследование примеси магния в кремнии
}

\author{
() Л.М. Порцель ${ }^{1}$, В.Б. Шуман ${ }^{1}$, А.А. Лаврентьев ${ }^{1}$, А.Н. Лодыгин ${ }^{1}$, Н.В. Абросимов ${ }^{2}$, Ю.А. Астров ${ }^{1, \uparrow}$ \\ ${ }^{1}$ Физико-технический институт им. А.Ф. Иофффе Российской академии наук, \\ 194021 Санкт-Петербург, Россия \\ ${ }^{2}$ Leibniz Institute for Crystal Growth, \\ 12489 Berlin, Germany \\ "E-mail: yuri.astrov@mail.ioffe.ru
}

Поступила в Редакцию 25 ноября 2019 г.

В окончательной редакции 5 декабря 2019 г.

Принята к публикации 5 декабря 2019 г.

\begin{abstract}
Измерены диффузионные профили концентрации электрически активной и полной концентрации примеси магния в кремнии. Диффузия проводилась сэндвич-методом в бестигельный бездислокационный кремний $n$-типа проволимости при температурах $T_{\text {diff }}=1000,1100^{\circ} \mathrm{C}$ и длительности процесса от 0.5 до 22.5 ч. Профили концентрации электрически активной компоненты магния $N_{\mathrm{Mg}_{i}}(x)$ определяли методом дифференциальной проводимости, профили полной концентрации $N_{\text {total }}(x)$ - методом вторично-ионной масс-спектроскопии. Установлено, что полная концентрация магния в образцах на $\sim 2$ порядка превосходит концентрацию электрически активной компоненты. Обнаружено также, что коэффициент диффузии межузельного магния, $D_{\mathrm{Mg}_{i}}$, зависит от времени диффузии и уменьшается при увеличении длительности процесса. Высказаны предположения о физических процессах, которые могут приводить к образованию электрически неактивной компоненты примеси магния и зависимости эффективного коэффициента диффузии от времени.
\end{abstract}

Ключевые слова: легирование кремния, диффузия, примесные центры, собственные дефекты.

DOI: $10.21883 /$ FTP.2020.04.49134.9318

\section{1. Введение}

Магний образует глубокие двухзарядные донорные центры в кремнии. Энергии уровней этих центров составляют $E_{c}-0.107$ эВ для нейтрального состояния $\mathrm{Mg}^{0}$ и $E_{c}-0.256$ эВ для $\mathrm{Mg}^{+}[1-3]$. В отличие от кремния с другими элементами II группы - Zn и Ве, атомы которых образуют двойные акцепторы и находятся в позициях замещения (substitution positions) в решетке кремния, электрическая активность $\mathrm{Si}: \mathrm{Mg}$ обусловлена атомами примеси, занимающими межузельные положения (tetrahedral interstitial positions) $\mathrm{Mg}_{i}[1,4]$.

Существуют различные способы легирования кремния магнием: в процессе выращивания кристалла $\mathrm{Si}$ из расплава [5], диффузия из газовой фазы [6,7], в процессе жидкофазной эпитаксии [8], ионная имплантация [9] и сэндвич-метод диффузии $[1,4]$. Основной объем информации о свойствах $\mathrm{Si}: \mathrm{Mg}$ получен с использованием образцов, легированных при высоких температурах с помощью сэндвич-метода. Этот метод позволяет получать объемно легированные образцы, концентрация магния в которых составляет $\sim 10^{15} \mathrm{~cm}^{-3}$. Рассматриваемая технология обычно предполагает закалку образцов после окончания процесса диффузии. В результате при низких температурах твердый раствор $\mathrm{Mg}_{i}$ в кристалле $\mathrm{Si}$ находится в термодинамически неравновесном состоянии, что в ряде случаев может приводить к изменению концентрации $\mathrm{Mg}_{i}$ со временем [10].

Установлено вместе с тем, что наряду с донорными центрами $\mathrm{Mg}_{i}$ в объеме кристалла присутствует и электрически неактивная компонента примеси. При этом полная концентрация магния в кристаллах значительно превосходит указанное выше значение концентрации доноров. Так, согласно работе [8], в образцах, изготовленных с помощью жидкофазной эпитаксии, концентрация магния достигала $\sim\left(10^{18}-10^{19}\right) \mathrm{cm}^{-3}$. Содержание примеси в цитируемой работе определялось с помощью атомно-абсорбционной спектроскопии. Близкие результаты получены также в [9], где в образцах $\mathrm{Si}$, легированных $\mathrm{Mg}$ методом ионной имплантации, исследовались профили полной концентрации и концентрации электрически активной примеси $\mathrm{Mg}$ в области аморфизации.

Несмотря на то что примесь магния в кремнии изучается уже в течение довольно длительного времени, технология получения $\mathrm{Si}: \mathrm{Mg}$ и свойства этого материала остаются мало изученными. В частности, только недавно была экспериментально определена температурная зависимость коэффициента диффузии межузельного магния $D_{\mathrm{Mg}_{i}}$ в бездислокационных кристаллах кремния [11-13]. $\mathrm{C}$ использованием оптических методов исследования в последнее время получен также ряд новых данных о свойствах центров $\mathrm{Mg}$ в кремнии [14-18].

В настоящей работе обнаружено, что диффузионное легирование кремния магнием с помощью сэндвичметода и последующей закалки образцов также сопровождается введением в объем кристалла как электрически активной компоненты, так и примеси в неактивном состоянии. Проведено экспериментальное исследование пространственных распределений обеих компонент, которые образуются в результате диффузии магния в 
бездислокационный кремний. Основное внимание уделяется изучению пространственных распределений при больших временах диффузии, которые обеспечивают проникновение примеси на значительные глубины.

\section{2. Методика экспериментов}

Для легирования магнием использовался выращенный в вакууме монокристаллический бестигельный бездислокационный кремний $n$-Si c удельным сопротивлением $\rho \approx 8.0 \cdot 10^{3} \mathrm{OM} \cdot \mathrm{cm}$ (концентрация кислорода $\sim 5 \cdot 10^{14} \mathrm{~cm}^{-3}$, углерода $\left.\sim 1 \cdot 10^{15} \mathrm{~cm}^{-3}\right)$. Диффузия примеси проводилась в пластины диаметром 30 мм и толщиной 1.8-2.0 мм. Источником диффузии служил слой $\mathrm{Mg}$ толщиной 1.2-1.5 мкм, который наносился на поверхность образцов путем распыления в вакууме металлического магния чистоты 99.995\%. В основной части опытов использовался режим односторонней диффузии, когда слой магния наносился только на одну сторону пластины. Затем со стороны слоя $\mathrm{Mg}$ к образцу прижималась вспомогательная пластинка кремния толщиной $\sim 0.5$ мм. Такая сэндвич-конструкция устанавливалась в кварцевую кассету, которая помещалась в кварцевую ампулу. Ампула заполнялась аргоном до некоторого давления и запаивалась. Основные опыты проводились на образцах, легированных при температуpax $T_{\text {diff }}=1000,1100^{\circ} \mathrm{C}$ и длительности процесса $t$ от 0.5 до 22.5 ч. При высокой температуре вспомогательная пластинка спаивалась с образцом, что препятствовало испарению магния. После окончания диффузии ампула охлаждалась в струе сжатого азота. Затем материал вспомогательной пластинки удалялся шлифовкой.

Как и в работах [11-13], распределения электрически активной компоненты магния по глубине определялись с использованием метода дифференциальной проводимости [19]. Для этого с поверхности образца последовательно удалялись слои толщиной $\sim 20$ мкм. Измерение сопротивления образца проводилось четырехзондовым методом [20] до и после удаления слоя.

С целью проверки данной методики определения профиля концентрации примеси был изготовлен образец $\mathrm{Si}: \mathrm{Mg}$ с заведомо однородным распределением примеси по глубине образца. Для этого в пластину толщиной 2 мм проводилась двухсторонняя диффузия магния при повышенной температуре, $T_{\text {diff }}=1250^{\circ} \mathrm{C}$, в течение 7.5 ч.

На рис. 1 приведены экспериментальные зависимости поверхностной проводимости от толщины обзразца, $\sigma_{s}(d)$, для образцов, полученных при $T_{\text {diff }}=1100^{\circ} \mathrm{C}$ и длительности процесса диффузии $t=2.5$ и 22.5 ч. На рисунке также показаны данные для образца, легированного с помощью двухсторонней диффузии. Близкое к линейному изменение поверхностной проводимости при уменьшении $d$, наблюдающееся для этого образца, свидетельствует о практически однородном распределении $\mathrm{Mg}_{i}$ в объеме образца.

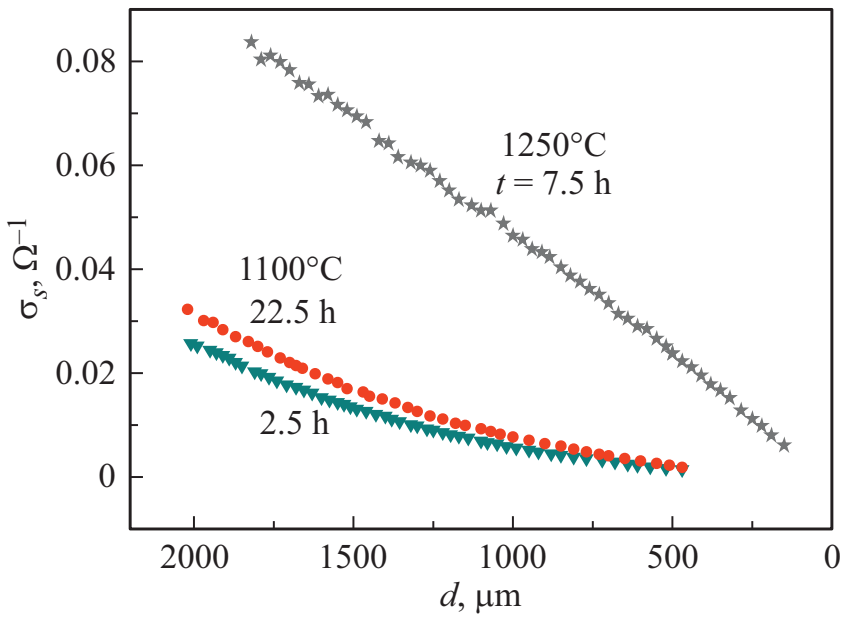

Рис. 1. Зависимости поверхностной проводимости $\sigma_{s}$ от толщины образца при последовательном удалении слоев материала.

Экспериментальные распределения $\sigma_{s}(d)$ аппроксимировались аналитическими функциями, которые использовались для расчета профилей концентрации свободных электронов $n$. Определение зависимостей концентрации электрически активной компоненты примеси магния от глубины проникновения $x, N_{\mathrm{Mg}_{i}}(x)$, проводилось на основе данных $n(x)$ и статистической модели двойного донора в кремнии, которая применялась в работах $[11,21]$ при обработке результатов измерений эффекта Холла.

Полученные распределения $N_{\mathrm{Mg}_{i}}(x)$ использовались для определения коэффициента диффузии $D_{\mathrm{Mg}_{i}}$. Для этого проводилась подгонка к экспериментальным кривым теоретических зависимостей, соответствующих модели диффузии из неограниченного источника:

$$
N_{\mathrm{Mg}_{i}}(x, t)=N_{S} \operatorname{erfc}\left(\frac{x}{2 \sqrt{D_{\mathrm{Mg}_{i} t}}}\right),
$$

где $N_{S}-$ концентрация примеси на поверхности, erfc - дополнительная функция ошибок Гаусса (Gauss complementary error function).

Распределения полной концентрации примеси магния $N_{\text {total }}(x)$ в кристаллах измерялись с помощью метода вторично-ионной масс-спектроскопии (ВИМС). В качестве зондирующего пучка использовались ионы $\mathrm{O}_{2}^{+} \mathrm{c}$ энергией 9.5 кэВ при токе $600 \mathrm{HA}$. Первичный пучок разворачивался в растр размером $500 \times 500$ мкм. Регистрировалась интенсивность сигнала положительно заряженных атомарных ионов Mg. Для устранения вклада ионов, распыленных со стенок кратера, в анализатор поступали ионы лишь из центра распыляемого кратера, ограниченного областью диаметром 120 мкм. Концентрация магния определялась путем сравнения с эталонным стандартом, полученным имплантацией магния в кремний. Минимальное значение концентрации $\mathrm{Mg}$, которое регистрировалось установкой, составляло $\sim 10^{16} \mathrm{~cm}^{-3}$. 
Для измерения распределений $N_{\text {total }}(x)$ методом ВИМС изготавливались образцы в форме клина. Для этого из пластин $\mathrm{Si}: \mathrm{Mg}$ вырезались образцы с размерами $10 \times 3 \times 2$ мм. Углы наклона клина $\alpha$ по отношению к начальной плоскости составляли 4,8 и $12^{\circ}$. Измерения концентрации $N_{\text {total }}$ осуществлялись последовательно в разных точках клина через равные интервалы $\Delta d \approx 1$ мм. Реализация метода позволяла определять распределения примеси до глубины образца, превышающей 1 мм.

\section{3. Экспериментальные результаты}

Были изготовлены две партии образцов, легированных при $T=1000$ и $1100^{\circ} \mathrm{C}$. Длительность диффузии для обеих партий составляла $t=0.5,1,2.5,7.5$ и 22.5 ч. Профили распределения концентрации магния $N_{\mathrm{Mg}_{i}}(x)$ для образцов, полученных при $T=1100^{\circ} \mathrm{C}$ и разных временах диффузии, представлены точками на рис. 2 (диффузионный профиль для $t=7.5$ ч расположен между распределениями для $t=2.5$ и 22.5 ч, не показан на рисунке).

На рисунке также представлен профиль концентрации магния $N_{\mathrm{Mg}_{i}}(x)$ в образце, полученном в результате двухсторонней диффузии при $T=1250^{\circ} \mathrm{C}$ в течение 7.5 ч. Наблюдается практически однородное распределение примеси в объеме образца. Эти данные подтверждают, таким образом, корректность методики, которая использовалась для измерения распределений на рис. 2, полученных в условиях односторонней диффузии.

Сплошными линиями на рисунке показаны зависимости согласно (1), наилучшим образом описывающие экспериментальные данные. Подгоночным параметром в расчетах служил коэффициент диффузии $D_{\mathrm{Mg}_{i}}$. Для образцов, легированных при $T=1000^{\circ} \mathrm{C}$, коэффициенты диффузии определялись аналогичным образом. Значения $D_{\mathrm{Mg}_{i}}$, соответствующие разным временам и температурам легирования, представлены в таблице.

Как выяснилось в ходе экспериментов, распределения концентрации $N_{\mathrm{Mg}_{i}}(x)$ и соответственно эффективные значения $D_{\mathrm{Mg}_{i}}$, определенные по описанной выше методике для времени диффузии 22.5 ч, зависят от толщины образца. По-видимому, это связано с влиянием условий рекомбинации собственных дефектов кремния на границе кристалла, удаленной от источника диффузии. Для коротких времен диффузии, $t=0.5-1$ ч, величины $D_{\mathrm{Mg}_{i}}$,

Значения коэффициента диффузии $D_{\mathrm{Mg}_{i}}$

\begin{tabular}{c|c|c}
\hline \multirow{2}{*}{ Время диффузии, ч } & \multicolumn{2}{|c}{$D_{\mathrm{Mg}_{i}},\left[\mathrm{~cm}^{2} \cdot \mathrm{c}^{-1}\right.$} \\
\cline { 2 - 3 } & $1000^{\circ} \mathrm{C}$ & $1100^{\circ} \mathrm{C}$ \\
\hline 0.5 & $3.0 \cdot 10^{-7}$ & $1.4 \cdot 10^{-6}$ \\
1.0 & $3.0 \cdot 10^{-7}$ & $8.0 \cdot 10^{-7}$ \\
2.5 & $2.5 \cdot 10^{-7}$ & $5.5 \cdot 10^{-7}$ \\
7.5 & $1.9 \cdot 10^{-7}$ & $2.2 \cdot 10^{-7}$ \\
22.5 & $1.2 \cdot 10^{-7}$ & $7.5 \cdot 10^{-8}$
\end{tabular}

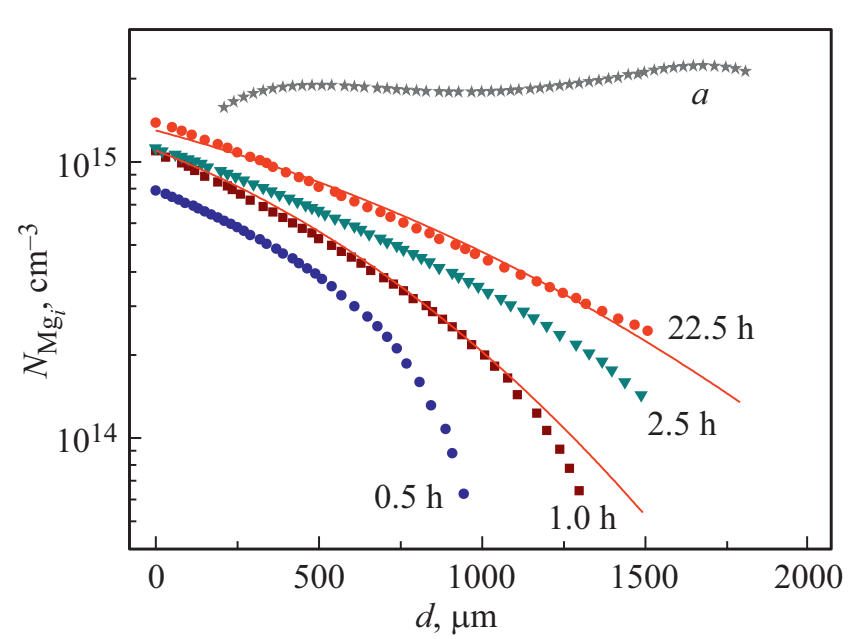

Рис. 2. Профили концентрации межузельного магния в образцах, легированных при $T=1100^{\circ} \mathrm{C}$ и различных длительностях диффузии. Сплошные кривые - примеры зависимостей, рассчитанных по формуле (1) с соответствующими коэффициентами диффузии (таблица). $a-$ профиль распределения примеси, полученный для случая диффузии магния с обеих сторон пластины при $T=1250^{\circ} \mathrm{C}$ и длительности процесса 7.5 ч.

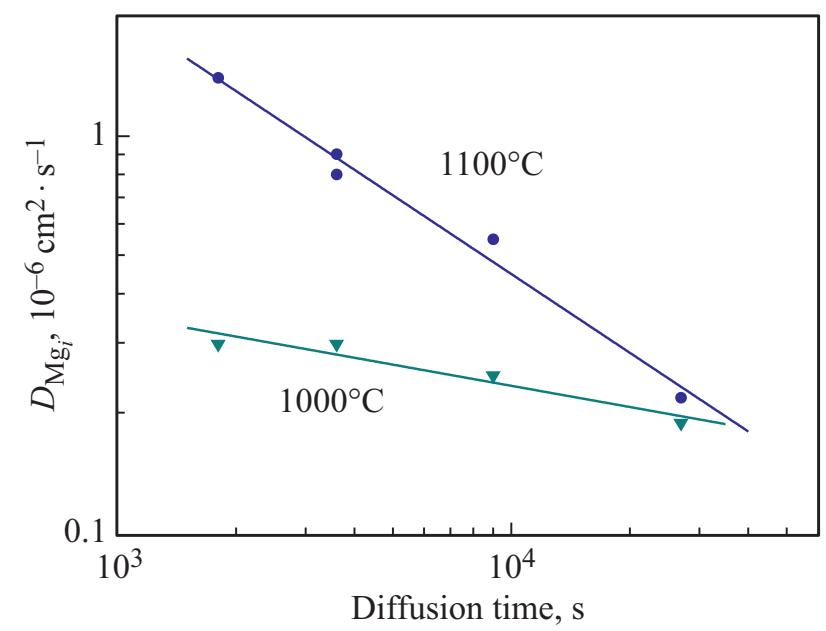

Рис. 3. Зависимости эффективного коэффициента диффузии межузельных центров магния от длительности диффузионного процесса при температурах 1000 и $1100^{\circ} \mathrm{C}$.

определенные в настоящей работе, соответствуют значениям $D_{\mathrm{Mg}_{i}}$, полученным нами ранее в работах [11-13].

Из данных таблицы следует, что эффективный коэффициент диффузии межузельного магния уменьшается с ростом $t$. На рис. 3 представлены соответствующие результаты для обеих температур процесса, которые в двойном логарифмическом масштабе хорошо аппроксимируются линейными зависимостями. Здесь не включены значения $D_{\mathrm{Mg}_{i}}$ для времени диффузии 22.5 ч.

На рис. 4 показаны профили полной концентрации магния $N_{\text {total }}(x)$, измеренные с помощью ВИМС, в образцах, легированных при длительностях диффузионного 


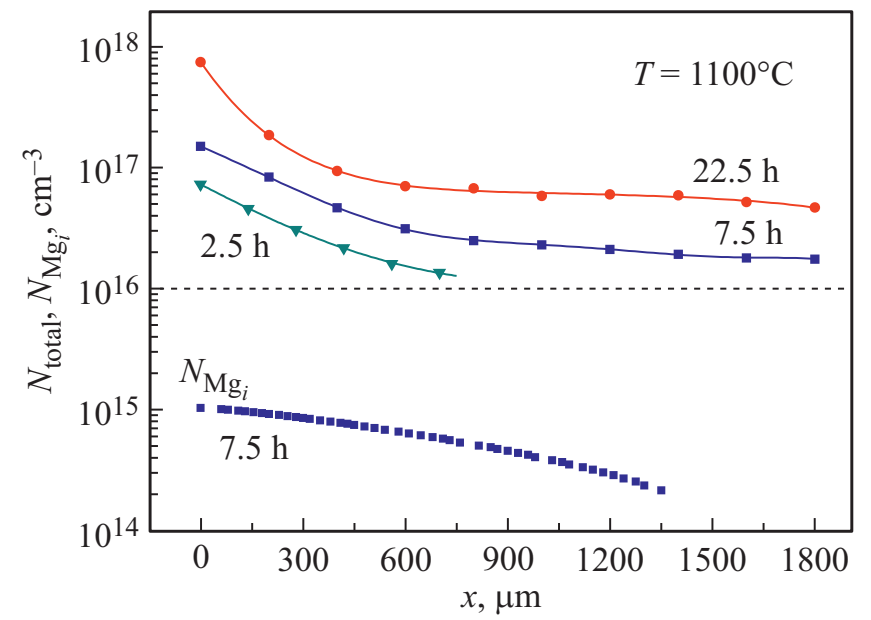

Рис. 4. Профили распределения полной концентрации $N_{\text {total }}(x)$ магния, измеренные методом ВИМС в образцах после диффузии в течение 2.5, 7.5 и 22.5 часов (верхние кривые) и профиль концентрации электрически активной компоненты $N_{\mathrm{Mg}_{i}}(x)$ для длительности диффузии 7.5 ч. Штриховая линия - нижний предел измерения экспериментальной установки.

процесса $t=2.5,7.5$ и 22.5 ч. Как отмечено выше, минимальная концентрация примеси, которая могла быть определена на нашей экспериментальной установке в наших условиях, составляет $\sim 10^{16} \mathrm{~cm}^{-3}$. Это значение отмечено на рис. 4 штриховой линией. Видно, что при длительной диффузии (22.5 ч) достигается значение полной концентрации $N_{\text {total }} \sim\left(10^{17}-10^{18}\right) \mathrm{cm}^{-3}$, и наблюдается почти однородное распределение магния по глубине образца.

Для иллюстрации разницы в пространственных распределениях обеих компонент в рис. 4 включен также профиль концентрации электрически активного магния $N_{\mathrm{Mg}_{i}}(x)$, полученный для времени диффузии 7.5 ч. В соответствии с этими данными значение $N_{\text {total }}$ почти на $\sim 2$ порядка превышает концентрацию электрически активных центров, которая достигается в этих условиях легирования.

\section{4. Обсуждение результатов}

Полученные данные свидетельствуют, таким образом, что в образцах монокристаллического $\mathrm{Si}$, легированных магнием с помощью сэндвич-метода диффузии и подвергнутых последующей закалке, наблюдаются следующие характерные особенности:

- в объем кристалла наряду с донорной примесью магния $\mathrm{Mg}_{i}$, вводится значительная концентрация примеси, которая не проявляет электрической активности $\left(N_{\text {total }}>N_{\mathrm{Mg}_{i}}\right)$;

- по мере увеличения длительности диффузии наблюдается замедление диффузионного распространения электрически активной компоненты примеси, которое формально соответствует снижению эффективного коэффициента диффузии;

- реальный коэффициент диффузии $\mathrm{Mg}$ в $\mathrm{Si}$ гораздо выше, чем эффективная величина, определяемая по профилю $N_{\mathrm{Mg}_{i}}(x)$.

Рассмотрим возможные процессы в кристалле, которые могут привести к наблюдаемым особенностям.

Наряду с $\mathrm{Mg}$ известен ряд других электрически активных примесей в кремнии, которые занимают в кристалле межузельные положения (interstitial positions), например, $\mathrm{Cu}$ [22], $\mathrm{Ni}$ [23], $\mathrm{Fe}$ [24]. Атомы этих элементов имеют высокую подвижность и могут образовывать комплексы с мелкими акцепторами и другими примесями, содержащимися в кристалле [25,26]. Такие комплексы являются электрически неактивными. Образование донорно-акцепторных пар приводит к уменьшению эффективного коэффициента диффузии $D_{\text {eff }}$ указанных примесей по сравнению с диффузией в достаточно чистом (близком к собственному) кремнии. Так, взаимодействие $\mathrm{Cu}$ с мелкими акцепторными примесями $\mathrm{B}, \mathrm{Al}, \mathrm{Ga}$ и In сопровождается образованием комплексов (например, пар $\mathrm{Cu}-\mathrm{Ga}[27]$ ), что приводит к уменьшению эффективного коэффициента диффузии $D_{\text {eff }}(\mathrm{Cu})$.

Как отмечено выше, исследования настоящей работы проводились с использованием монокристаллического бестигельного бездислокационного кремния высокой чистоты. Можно считать, что образование электрически неактивной компоненты примеси магния нельзя объяснить взаимодействием $\mathrm{Mg}$ с другими примесями или дефектами, которые могут существовать в кристалле, аналогично тому, как это имеет место в случае кремния, легированного $\mathrm{Cu}$ или $\mathrm{Ni}$.

Предположение о том, что электрически неактивные состояния магния в кристалле представляют собой донорно-акцепторные пары, было выдвинуто в работе [9]. По мнению автора, основная часть магния в кристалле содержится в виде электрически нейтральных пар, которые состоят из атома магния, расположенного в узле решетки кремния, (substitutional) $\mathrm{Mg}_{s}$, и межузельного (interstitial) атома $\mathrm{Mg}_{i}$. Согласно $[9,28]$, такие комплексы $\left(\mathrm{Mg}_{s}-\mathrm{Mg}_{i}\right)$ могут образовываться в результате взаимодействия отрицательно заряженных при температуре диффузии $\mathrm{Mg}_{s}$, которые являются акцепторами, и положительно заряженных $\mathrm{Mg}_{i}$.

Существование таких пар было убедительно доказано в статье [17], однако концентрация таких центров не оценивалась.

Комплексы $\left(\mathrm{Mg}_{s}-\mathrm{Mg}_{i}\right)$ могут распадаться вследствие вытеснения узельного $\mathrm{Mg}_{s}$ в межузельные положения собственными межузельными атомами кремния (selfinterstitials) $I$, согласно механизму kick-out [29]. Дефекты $\mathrm{Mg}_{i}$ диффундируют по междоузлиям и в процессе движения могут переходить в узлы решетки, выталкивая атомы кремния в междоузлия. Узельные $\mathrm{Mg}_{s}$ могут снова образовывать пары $\left(\mathrm{Mg}_{s}-\mathrm{Mg}_{i}\right)$.

В рамках модели kick-out можно пытаться найти объяснение зависимости эффективного коэффициента 
диффузии межузельных атомов примеси $X_{i}$ от времени процесса. Диффузия таких атомов в бездислокационном кремнии может приводить к возникновению пересыщения кристалла собственными межузельными атомами I. Отклонение пространственного распределения собственных дефектов от равновесного значения устанавливается в самом начале диффузионного процесса. С течением времени пересышение $I$ в объеме кристалла уменьшается в результате диффузии межузельных атомов кремния к поверхностям, которые являются стоком для них [29]. Участие собственных межузельных атомов в обмене $X_{i} \leftrightarrow X_{s}+I$ может привести к тому, что с уменьшением концентрации $I$ увеличивается скорость образования узельных атомов $X_{s}$. Это, в свою очередь, вызовет замедление движения атомов $X_{i}$ и соответственно уменьшение эффективного коэффициента диффузии $D_{\text {eff. }}$

Нам не удалось найти в литературе экспериментальных данных о зависимости эффективного коэффициента диффузии от времени для других быстродиффундирующих примесей в кремнии, которые могут занимать межузельные положения. Отметим вместе с тем, что косвенным свидетельством существования таких эффектов является анализ диффузионных процессов в $\mathrm{Si}: \mathrm{Zn}$, проведенный в $[29,30]$.

К сожалению, ввиду недостаточности экспериментальных данных трудно определить природу нейтральной компоненты примеси магния и выявить физические процессы, связанные с ее образованием. По-видимому, это могут быть как пары $\left(\mathrm{Mg}_{s}-\mathrm{Mg}_{i}\right)$, так и атомы магния в позиции замещения, $\mathrm{Mg}_{s}$. Кроме того, электрически нейтральные атомы магния могут содержаться в кристалле в виде преципитатов $\mathrm{Mg}_{2} \mathrm{Si}$ или других образований [31]. В любом случае формирование нейтральной компоненты примеси $\mathrm{Mg}$ происходит в результате диффузии межузельных атомов магния $\mathrm{Mg}_{i}$, которая определяется квазихимическими реакциями с участием собственных дефектов кремния. Замедление диффузии $\mathrm{Mg}_{i}$ со временем можно связать с возрастанием концентрации центров, которые взаимодействуют с межузельным магнием, снижая тем самым подвижность атомов в процессе их распространения в объеме кристалла.

\section{5. Заключение}

Таким образом, в работе установлено, что при легировании пластин кремния магнием с помощью сэндвичметода основная часть примеси в образцах находится в электрически неактивном состоянии. Обнаружено также, что с увеличением времени диффузии наблюдается уменьшение коэффициента диффузии межузельного магния. Эта особенность проявляется для двух исследованных температур диффузии, 1000 и $1100^{\circ} \mathrm{C}$, причем эффект выражен сильнее при $T=1100^{\circ} \mathrm{C}$. Высказаны предположения о физических процессах, которые ответственны за наблюдаемые явления.

\section{Благодарности}

Авторы благодарят А.С. Милентьева за помощь в экспериментах.

\section{Конфликт интересов}

Авторы заявляют, что у них нет конфликта интересов.

\section{Список литературы}

[1] L.T. Ho, A.K. Ramdas. Phys. Rev. B, 5, 462 (1972).

[2] L.T. Ho. Phys. Status Solidi B, 81, K99 (1977).

[3] L.T. Ho. Def. Dif. Forum, 221-223, 41 (2003).

[4] R.F. Franks, J.B. Robertson. Solid State Commun., 5, 479 (1967).

[5] N.V. Abrosimov, N. Nötzel, H. Riemann, K. Irmscher, S.G. Pavlov, H.-W. Hübers, U. Böttger, P.M. Haas, N. Drichko, M. Dressel. Sol. St. Phenomena, 131-133, 589 (2008).

[6] E. Ohta, M. Sakata. Solid State Electron., 22, 677 (1979).

[7] K. Matsumoto, Y. Uenaka, Y. Seto, H. Yashiro, H. Nakamura, T. Kimura, T. Uchino. J. Appl. Phys., 108, 113706 (2010).

[8] H. Sigmund. J. Electrochem. Soc., 129 (12), 2809 (1982).

[9] H. Sigmund, D. Weiß. In: Ion Implantation: Equipment and Techniques, ed. by H. Ryssel, H. Glawishing [Springer Ser. Electrophysics, 11, 473 (1983)].

[10] L.T. Ho. Phys. Status Solidi A, 28, K73 (1975).

[11] Yu.A. Astrov, V.B. Shuman, L.M. Portsel, A.N. Lodygin, S.G. Pavlov, N.V. Abrosimov, V.N. Shastin, H.-W. Hübers. Phys. Status Solidi A, 214 (7), 1700192 (2017).

[12] V.B. Shuman, A.A. Lavrent'ev, Yu.A. Astrov, A.N. Lodygin, L.M. Portsel. Semiconductors, 51 (1), 1 (2017).

[13] V.B. Shuman, Yu.A. Astrov, A.N. Lodygin, L.M. Portsel. Semiconductors, 51 (8), 1031 (2017).

[14] S.G. Pavlov, N.V. Abrosimov, V.B. Shuman, L.M. Portsel, A.N. Lodygin, Yu.A. Astrov, R.Kh. Zhukavin, V.N. Shastin, K. Irmscher, A. Pohl, H.-W. Hübers. Phys. Status Solidi B, 256 (6), 1800514 (2019).

[15] S.G. Pavlov, N. Dessmann, A. Pohl, V.B. Shuman, L.M. Portsel, A.N. Lodygin, Yu.A. Astrov, S. Winnerl, H. Schneider, N. Stavrias, A. van der Meer, V.V. Tsyplenkov, K.A. Kovalevsky, R.Kh. Zhukavin, V.N. Shastin, N.V. Abrosimov, H.-W. Hübers. Phys. Rev. B, 94, 075208 (2016).

[16] R.J.S. Abraham, V.B. Shuman, L.M. Portsel, A.N. Lodygin, Yu.A. Astrov, N.V. Abrosimov, S.G. Pavlov, H.-W. Hübers, S. Simmons, M.L.W. Thewalt. Phys. Rev. B, 99, 195207 (2019).

[17] R.J.S. Abraham, A. DeAbreu, K.J. Morse, V.B. Shuman, L.M. Portsel, A.N. Lodygin, Yu.A. Astrov, N.V. Abrosimov, S.G. Pavlov, H.-W. Hübers, S. Simmons, M.L.W. Thewalt. Phys. Rev. B, 98, 205203 (2018).

[18] R.J.S. Abraham, A. DeAbreu, K.J. Morse, V.B. Shuman, L.M. Portsel, A.N. Lodygin, Yu.A. Astrov, N.V. Abrosimov, S.G. Pavlov, H.-W. Hübers, S. Simmons, M.L.W. Thewalt. Phys. Rev. B, 98, 045202 (2018).

[19] B.I. Boltaks. Diffusion in Semiconductors (Academic, N. Y., 1963).

[20] F.M. Smits. Bell Syst. Techn. J., 37, 711 (1958).

[21] Yu.A. Astrov, L.M. Portsel, A.N. Lodygin, V.B. Shuman. Semicond. Sci. Technol., 26, 055021 (2011). 
[22] A.A. Istratov, E.R. Weber. J. Electrochem. Soc., 149 (1), G21 (2002).

[23] J. Lindroos, D.P. Fenning, D.J. Backlund, E. Verlage, A. Gorgulla, S.K. Estreicher, H. Savin, T. Buonassisi. J. Appl. Phys., 113, 204906 (2013).

[24] A.A. Istratov, H. Hieslmair, E.R. Weber. Appl. Phys. A, 69, 13 (1999).

[25] E. Weber. Appl. Phys. A, 30, 1 (1983).

[26] C.A. Wert, R.C. Frank. Ann. Rev. Mater. Sci., 13, 139 (1983).

[27] A.A. Istratov, C. Flink, H. Hieslmair, E. Weber, T. Heiser. Phys. Rev. Lett., 81 (6), 1243 (1998).

[28] N. Baber, L. Montelius, M. Kleverman, K. Bergman, H.-G. Grimmeiss. Phys. Rev. B, 38 (15), 10483 (1988).

[29] U. Gösele, W. Frank, A. Seeger. J. Appl. Phys., 23, 361 (1980).

[30] H. Bracht, N.A. Stolwijk, H. Mehrer. Phys. Rev. B, 52 (23), 16542 (1995).

[31] H. Bracht, N.A. Stolwijk, I. Yonenaga, H. Mehrer. Phys. Status Solidi A, 137, 499 (1993).

Редактор Л.В. Шаронова

\section{Study of magnesium impurity in silicon}

L.M. Portsel ${ }^{1}$, V.B. Shuman ${ }^{1}$, A.A. Lavrent'ev' ${ }^{1}$, A.N. Lodygin ${ }^{1}$, N.V. Abrosimov ${ }^{2}$, Yu.A. Astrov ${ }^{1}$

${ }^{1}$ loffe Institute, 194021 St. Petersburg, Russia

${ }^{2}$ Leibniz Institute for Crystal Growth, 12489 Berlin, Germany

Abstract Profiles of both electrically active and total concentration of magnesium impurity in silicon were measured. Diffusion was performed into the floating zone dislocation-free $n$-type silicon at the temperatures $T_{\mathrm{diff}}=1000,1100^{\circ} \mathrm{C}$ and duration from 0.5 to $22.5 \mathrm{~h}$. The depth profiles of the electrically active interstitial magnesium concentration $N_{\mathrm{Mg}_{i}}(x)$ were determined by differential conductivity method, while the total concentration profile $N_{\text {total }}(x)$ - with secondary ion mass spectroscopy. The total concentration of magnesium was found to be almost two orders higher than the concentration $N_{\mathrm{Mg}_{i}}$. It turned out that the effective diffusion coefficient $D_{\mathrm{Mg}_{i}}$ decreases with the diffusion time. Possible physical processes responsible for formation of the electrically inactive component of magnesium impurity and the dependence of effective diffusion coefficient on time are discussed. 